\title{
Sistema de classificação de pacientes na
especialidade enfermagem psiquiátrica*
}

\author{
Patient system classification in psychiatric nursing
}

Sistema de clasificación de los pacientes de la especialidad enfermería psiquiátrica

\author{
Paula Andréa Shinzato Ferreira Martins', Hideko Takeuchi Forcella²
}

\begin{abstract}
RESUMO
Objetivo: Estudar um Instrumento para Classificação do Nível de Dependência de Pacientes Psiquiátricos aos Cuidados de Enfermagem, foi o objetivo deste estudo. Métodos: Os indicadores críticos do cuidado (aparência e higiene, expressão do pensamento, humor e afeto, interação social, atividades, alimentação e hidratação, sono, medicação, eliminações, sinais vitais e outros controles, problemas e queixas somáticas). Resultados: com gradação de 1 a 3 pontos permitiram identificar os níveis de dependência. Foi aplicada a técnica Delphi e, os avaliadores concordaram pela manutenção dos 11 indicadores críticos. Conclusão: Os resultados deste estudo mostram que o instrumento é válido e pode contribuir para o avanço tecnológico e científico da Especialidade no Brasil.
\end{abstract}

Descritores: Enfermagem psiquiátrica; Cuidados de enfermagem.

\begin{abstract}
Objective: The aim of this paper was to report the validity of an Instrument for Classification of Nursing Care Dependency Levels for Psychiatric Patients. Methods: The development of the instrument was based on the following critical indicators of care: appearance and hygiene, thought expression, humor and affection, social interaction, activities, feeding and hydration, sleep, medication, eliminations, vital signs and others controls, and problems and somatic complaints. Results: The scores for degree of dependence are graded on a 3-point scale which permits to determine dependency levels. The Delphi technic was used to validate the instrument. Experts agreed with the maintenance of 11 critical indicators. Conclusion: The results of this study showed the instrument is valid and may contribute to technological and scientific advances for psychiatric speciality in Brazil.
\end{abstract}

Keywords: Psychiatric nursing; Nursing care.

\section{RESUMEN}

Objetivos: Estudiar un Instrumento de Clasificación del Nivel de Dependencia de Pacientes Psiquiátricos bajo los Cuidados de Enfermería. Métodos: Los indicadores críticos del cuidado (apariencia e higiene, expresión del pensamiento, humor y afecto, interacción social, actividades, alimentación e hidratación, sueño, medicación, eliminaciones, signos vitales y otros controles, problemas y quejas somáticas). Resultados: con graduación de 1 a 3 puntos permitieron identificar los niveles de dependencia. Fue aplicada la técnica Delphi, concordando los evaluadores por la manutención de los 11 indicadores críticos. Conclusión: Aos resultados mostran que el instrumento es valido e puede contribuir al avance tecnológico y científico de la especialidad en Brasil.

Descriptores: Enfermería psiquiátrica; Cuidados de enfermería.

\footnotetext{
* Resumo de Dissertação de Mestrado apresentada à Escola de Enfermagem da Universidade de São Paulo - SP - São Paulo (SP) - Brasil ${ }^{1}$ Especialista e Mestre em Enfermagem Psiquiatrica e Saúde Mental. Docente do Curso de Graduação em Enfermagem da Universidade de Sant'Anna (SP), Brasil.

${ }^{2}$ Doutora em Enfermagem. Orientadora do Programa de Pós-Graduação da Escola de Enfermagem da Universidade de São Paulo (SP), Brasil.
} 


\section{INTRODUÇÃO}

As novas práticas assistenciais, intra e extrahospitalares, no campo da saúde mental exigem remodelações ou adaptações no âmbito gerencial da enfermagem psiquiátrica. Embora muito se tenha falado sobre estas novas práticas e das políticas de saúde mental, poucas são as referências sobre o cálculo de profissionais necessários para assistir com qualidade um paciente psiquiátrico e a quantos pacientes um profissional consegue atender terapeuticamente.

Atualmente existem meios que favorecem aos enfermeiros pesquisadores o acesso e o intercâmbio de informações, o quê possibilitaria a realização de estudos sobre o tema dimensionamento de pessoal em enfermagem psiquiátrica, para isso são necessários recursos financeiros, investimentos das instituições e integração dos serviços e academia ${ }^{(1)}$. A escassez de publicações sobre o tema motivou a realização deste estudo.

Entretanto, para dimensionar a equipe de enfermagem, segundo a literatura, há um trajeto a ser percorrido, e este se inicia com um Sistema de Classificação de Pacientes (SCP), cujo conceito surgiu da exigência, das organizações de saúde de racionalizar o trabalho e conseqüentemente, os recursos humanos e materiais ${ }^{(2)}$.

A classificação de pacientes existe desde o período de Florence Nightingale que procurava organizar as enfermarias de modo que os mais graves fossem alocados nas proximidades das mesas das enfermeiras ${ }^{(2)}$.

Através de um estudo desenvolvido em hospitais de Nova York, em 1930, foi encontrado um indicador de horas para as necessidades do paciente, que originou o SCP. Apesar deste indicador não ser exato, o mesmo ofereceria subsídios para outros estudos posteriores ${ }^{(3)}$.

No Brasil, em 1950, um estudo possibilitou a determinação das necessidades de cuidados de enfermagem para pacientes das clínicas médicocirúrgicas $^{(3)}$, originando a noção de classificação de pacientes.

No final da década de setenta, surgiram proposta de parâmetros para ajustar as tabelas de lotação do pessoal no serviço de enfermagem, adotando assim seis elementos para compor o cuidado progressivo em enfermagem ${ }^{(4)}$. Neste estudo, a enfermagem psiquiátrica não foi contemplada, talvez por não fazer parte da realidade operacional das unidades atendidas pela proposta ou por dissolver-se entre as demais especialidades.

No entanto, a chamada "classificação" de pacientes, de acordo com suas necessidades já vinha sendo utilizada pelos japoneses há mais de cem anos, segundo dados do mesmo estudo ${ }^{(4)}$.

$\mathrm{Na}$ literatura, são encontradas várias definições para o SCP: "um processo no qual se procura categorizar pacientes de acordo com a quantidade de cuidado de enfermagem requerido, ou seja, baseado na complexidade da assistência de enfermagem"(5), "uma forma de determinar o grau de dependência de um paciente em relação à equipe de enfermagem"(()), e "um instrumento indispensável à gerência de enfermagem"(4).

Dos trabalhos sobre SCP no Brasil e no mundo podemos destacar que ao longo dos anos foram sendo desenvolvidos sistemas com finalidades distintas, tais como organizar o serviço de enfermagem ${ }^{(3-5,7-15)}$; melhorar a qualidade do serviço de enfermagem ${ }^{(2-}$ 4,16) e determinar os custos de enfermagem ${ }^{(17)}$.

Os cálculos da força de trabalho de enfermagem devem ser efetuados segundo a complexidade dos pacientes, no entanto as classificações existentes referemse àqueles pacientes internados em hospitais gerais que necessitam de cuidados físicos em maior ou menor grau de dependência ${ }^{(14)}$.

Para determinar o número de profissionais, a melhor estratégia inclui o reconhecimento do perfil da população a ser atendida, o grau de dependência da enfermagem e ou o nível de auto-suficiência do paciente ${ }^{(18)}$.

Cabe ainda ressaltar que o sistema de classificação de pacientes é considerado "sistema", pelas várias partes que se integram, não sendo utilizado apenas para viabilizar o dimensionamento de enfermagem, mas também como indispensável para a oferta de assistência de maior qualidade, planejamento de planta física do serviço, alocação de pessoal, entre outros.

$\mathrm{Na}$ tentativa de amparar o enfermeiro no estabelecimento do quadro quantitativo de profissionais, o COFEN - Conselho Federal de Enfermagem, em 1996, na "Resolução COFEN-189 estabelece parâmetros para Dimensionamento do Quadro de Profissionais", apresentando considerações sobre a responsabilidade do enfermeiro pela confecção desse quadro de profissionais ${ }^{(16)}$.

O referencial utilizado pela Resolução foi adaptado do trabalho de Fugulin et al, realizado em 1994, e estabelece categorias de pacientes por complexidade assistencial dividindo-as em: assistência mínima/ autocuidado, assistência intermediária, assistência semiintensiva e assistência intensiva. Ressalte-se que os aspectos psíquicos não são abordados, restringindo-se às exigências evidenciadas pelos atendimentos das necessidades humanas básicas. Inclusive as classificações existentes são de difícil aplicação em pacientes psiquiátricos, visto que não atendem às suas particularidades.

Os pacientes psiquiátricos apresentam circunstâncias que requerem plena assistência de enfermagem, como por exemplo, o uso inadequado de roupas e adornos, a presença de comportamentos alterados, tentativa de fuga, ideação e tentativa de suicídio, potencial para auto e heteroagressividade, entre outros. Nestas circunstâncias 
eles requerem assistência "semi-intensiva" e ou "intensiva" embora, nem sempre haja "risco iminente de vida, sujeitos à instabilidade de funções vitais", segundo a Resolução COFEN - 189 $9^{(16)}$.

Além disso, é possível notar que na enfermagem psiquiátrica, muitas vezes, o paciente não apresenta nenhuma alteração clínica não psiquiátrica, precisando porém, de outrem para orientar-estimular-supervisionar suas atividades e cuidados de vida diária, aspectos não abordados nas categorias propostas pela Resolução.

Por outro lado, nas unidades de internação psiquiátrica, vivenciam-se situações específicas como pacientes inquietos e agitados que necessitam de espaço tranqüilo e seguro, com a presença de profissionais que saibam estabelecer limites com flexibilidade, tolerância e firmeza e neste contexto, evidenciam-se, ainda, dois conflitos, um que é numérico - quantos profissionais? $\mathrm{Um}$, dois, ... $\mathrm{E}$ se o paciente tornar-se heteroagressivo? Serão necessários cinco elementos para atender com segurança a intercorrência; e outro que se refere ao treinamento e capacitação específica - todo enfermeiro, técnico de enfermagem (TE) e auxiliar de enfermagem (AE) estão capacitados para atender pacientes com flexibilidade, tolerância e firmeza? E se firmeza for "confundida" com rigidez? Pacientes com potencial para tentativa de suicídio, necessitam de observação rigorosa, para se detectar seu real estado. Torna-se necessário capacitar membros da equipe de enfermagem no que concerne à honestidade da relação estabelecida, garantindo flexibilidade, tolerância, firmeza e afeto.

O afeto é aspecto essencial na relação - enfermeiro/ TE/AE - paciente-, pois este tipo de relacionamento deve estar pautado nas emoções e no vínculo profissional, não, em vínculos de "amizade" e "coleguismo". Como garantir tudo isso?

Pacientes psiquiátricos em fase aguda da doença, colocam em risco seu próprio bem-estar, proteção e integridade e podem expor os demais (outros pacientes, familiares e profissionais) a riscos.

Desde 1982, alguns pesquisadores desenvolvem estudos, procurando testar, validar e reavaliar SCP psiquiátricos, por intermédio de medição do tempo investido por atividade e outras categorias ${ }^{(19)}$, já os SCP apresentados para enfermagem psiquiátrica não foram considerados abrangentes por não considerar pacientes em fase aguda dos transtornos ${ }^{(18)}$ e, mesmo que SCP gerais possam ser adaptados para hospitais psiquiátricos, há de se desenvolver instrumentos específicos para classificar estes pacientes ${ }^{(19)}$.

A vivência prática tem mostrado que o número de profissionais que estão envolvidos nas atividades de cuidar ou assistir o doente mental, bem como suas qualificações encontram-se diretamente relacionadas ao melhor desempenho da equipe e a melhora do quadro psiquiátrico do paciente internado.

A quantificação ou a determinação de horas de prestação de serviços em enfermagem psiquiátrica necessita de dimensionamento peculiar, quando comparada às horas gastas nas unidades gerais e, até o presente momento, este aspecto ainda não foi estudado.

Considerando o exposto, este estudo teve a proposta de desenvolver um instrumento para classificação do nível de dependência de pacientes psiquiátricos aos cuidados de enfermagem, testando-o com a clientela em fase aguda do transtorno psíquico, usuária de unidades de internação.

\section{OBJETIVOS}

O objetivo geral deste estudo foi o de construir um sistema de classificação de pacientes com transtornos psiquiátricos, a fim de colaborar com a melhoria da qualidade da assistência e com o desenvolvimento técnicocientífico da enfermagem psiquiátrica.

Os objetivos específicos foram os de: a) identificar os indicadores críticos essenciais à classificação de pacientes com transtornos psiquiátricos; b) elaborar um instrumento de classificação de pacientes, baseado nas necessidades individualizadas do cuidado de enfermagem ao paciente com transtorno psíquico; c) validar o conteúdo do instrumento elaborado para a especialidade.

\section{MÉTODOS}

Trata-se de um estudo descritivo. Foram sujeitos da pesquisa, sete enfermeiros especialistas em enfermagem psiquiátrica e saúde mental que fizeram o julgamento do instrumento elaborado e proposto, levando em consideração o conhecimento e a vivência destes na área especializada.

O trajeto metodológico percorrido refere-se às etapas de construção e desenvolvimento de um instrumento para classificação de pacientes da especialidade enfermagem psiquiátrica, da validação do instrumento e do estabelecimento da padronização dos níveis de dependência desses pacientes.

Para elaborar um sistema de classificação de pacientes, foi necessário desenvolver um instrumento capaz de demonstrar os diferentes níveis de dependência.

$\mathrm{O}$ instrumento desenvolvido para esse estudo seguiu o modelo de sistemas de avaliação de indicadores e, assim, foram estabelecidos indicadores para promoção do cuidado de pacientes adultos, atendidos em unidades de internação psiquiátrica, com distintos diagnósticos clínicos na fase aguda ou de melhora dos transtornos e ainda em situação de pré-alta, quando apresentavam condições de se manterem em suas atividades habituais.

$\mathrm{O}$ instrumento proposto denominado "Classificação do Nível de Dependência para 
Enfermagem Psiquiátrica" foi composto por 11 indicadores críticos do cuidado, cada um com gradação de 1 a 3 pontos, tendo o objetivo específico de identificar o nível de dependência dos pacientes atendidos quanto à intensidade crescente da complexidade da assistência necessária.

Os indicadores críticos relacionados no instrumento e suas denominações foram elaborados, baseando-se no Roteiro de "Observação de Comportamento de pacientes internados em hospital psiquiátrico" proposto por Arantes ${ }^{(20)}$; nos modelos de instrumentos e sistemas já estudados por Perroca ${ }^{(5)}$ e Romero ${ }^{(11)}$, bem como, na experiência da autora do estudo.

Os indicadores foram: cuidados com a aparência e higiene, expressão do pensamento, humor, interação social, atividades, alimentação e hidratação, sono, medicação, eliminações, sinais vitais e outros controles, problemas e queixas somáticas.

Conforme citado anteriormente, cada indicador apresentou três variáveis de nível de dependência: discreta, intermediária e plena aos cuidados da enfermagem psiquiátrica.

\section{Classificação por Nível de Dependência}

\begin{tabular}{ll} 
Pontuação & \multicolumn{1}{c}{ Descrição } \\
11 a 18 & Grau de Dependência Discreta \\
19 a 26 & Grau de Dependência Intermediária \\
27 a 33 & Grau de Dependência Plena
\end{tabular}

Considerando os 11 indicadores críticos estabelecidos e o valor atribuído a cada um deles, a variação de pontuação foi de 11 a 33 pontos. Assim, optou-se por distribuir os pontos em três categorias com intervalos de classe a cada 8 pontos.

Uma vez elaborado um instrumento para classificar o nível de dependência de pacientes aos cuidados de enfermagem psiquiátrica, tornou-se imprescindível sua validação.

A validação do instrumento desenvolvido foi realizada pela autora do estudo, utilizando a técnica Delphi.

Tomando-se em consideração que validade referiase "à precisão do instrumento em medir o que se propõe a medir", logo, "para que um instrumento fosse considerado válido seria necessário que fosse confiável”(5). Para validar o instrumento proposto, optou-se por consultar a opinião de enfermeiros com vasta experiência profissional, somente para a validação do conteúdo do instrumento, uma vez que a validação clínica, deverá ser objeto de estudo posterior. Estes enfermeiros compuseram o "painel" e foram denominados "juízes especialistas".

Deste modo inicialmente, os elementos participantes do "grupo de juízes especialistas" também denominado "painel de juízes especialistas" foram identificados levando-se em consideração a experiência na área específica.

Os critérios estabelecidos para inclusão de enfermeiros no quadro de juízes especialistas foram, ser enfermeiro com título de especialista e ou com curso de pósgraduação - mestre e ou doutor - em Enfermagem Psiquiátrica e Saúde Mental; ter cinco anos ou mais de experiência em Enfermagem Psiquiátrica e Saúde Mental em instituições de ensino (graduação e pós-graduação) e ou de assistência ao portador de transtornos mentais intra e ou extra-hospitalar; e aceitar participar do estudo na condição de juiz especialista.

Todos os enfermeiros contatados consentiram em participar deste estudo.

A técnica Delphi utilizada por Spínola ${ }^{(21)}$ não estabelece o número ideal de informantes sendo, o diferencial, a especialização de cada um.

Posteriormente, os questionários foram elaborados para identificar os juízes em sua formação e atuação profissional; para obter o consentimento livre e informado daqueles que aceitaram participar do estudo, após orientações sobre o mesmo; para julgar, por intermédio de perguntas fechadas e abertas, individualmente, cada item em sua pertinência e, tecer comentários e sugestões.

A coleta dos dados foi realizada pessoalmente pela autora. Após o recebimento das respostas dos juízes, realizaram-se a análise e as súmulas dos resultados.

Em cada etapa do estudo Delphi, ocorreu a análise parcial dos resultados, a busca de convergência das respostas e a coerência entre os juízes para determinar o melhor instrumento para a classificação dos pacientes.

Nesta pesquisa, houve consenso entre os juízes com apenas uma rodada de correspondência. De acordo com a Técnica Delphi, o nível de consenso do grupo de juízes especialistas é reservado ao investigador, sendo aceitáveis níveis entre 50 e $80 \%{ }^{(22)}$. Para o presente estudo, a pesquisadora adotou desde o início, o nível de 70,0\% ou mais de consenso para cada item validado do instrumento elaborado.

Foi evidenciado que o painel de juízes especialistas consideraram pertinente os 11 indicadores críticos desenvolvidos.

O nível de consenso variou entre $86,0 \%$ e $100,0 \%$, considerando que o adotado foi maior ou igual a 70,0\% e que se obteve um mínimo de $86,0 \%$.

Todos os indicadores críticos construídos foram considerados validados, logo, compuseram o instrumento para classificação de pacientes psiquiátricos quanto ao grau de dependência aos cuidados de enfermagem.

As contribuições dos juízes acerca de cada indicador foram de grande valia. Alguns sugeriram alterações de palavras, a fim de melhorar a concordância e ou 
enriquecer o conteúdo com aspectos mais abrangentes.

Cabe ressaltar a tendência à heterogeneidade dos juízes, já prevista pela técnica Delphi, no que se refere ao emprego de termos técnicos, expressões profissionais e crenças pessoais sobre a enfermagem psiquiátrica, o paciente atendido e todo o cenário deste atendimento.

Ainda, foram avaliados os demais itens, quanto a sua pertinência, como: "Totalização dos Pontos", "Diagnóstico (médico)", identificação da data, período do dia e enfermeiro responsável pelo preenchimento e "classificação por nível de dependência".

Ressaltamos que a validação do instrumento desenvolvido para classificar pacientes quanto ao grau de dependência aos cuidados de enfermagem psiquiátrica abrange todo o instrumento, logo, os dados complementares também foram validados e o consenso obtido variou entre $86,0 \%$ e $100,0 \%$.

Sobre a pertinência da denominação de cada categoria dos níveis de dependência todos os juízes aprovaram, na sua totalidade, as denominações: discreta, intermediária e plena, propostas pelo instrumento.

O "Instrumento para Classificação do Nível de Dependência em Enfermagem Psiquiátrica", é apresentado, a seguir, em sua versão final validada.

\section{CONCLUSÕES}

Os indicadores críticos essenciais à classificação dos pacientes foram identificados e o instrumento de classificação foi elaborado com base nas necessidades individualizadas do cuidado de enfermagem ao paciente com transtorno psiquiátrico.

O conteúdo do instrumento específico foi validado. Os dados coletados na primeira rodada de correspondência evidenciaram consenso maior do que o determinado no estudo, ou seja, $70,0 \%$ ou mais de consenso entre os juízes especialistas para cada item. A validade foi obtida baseada nas opiniões de sete enfermeiros, juízes especialistas que concordaram com a manutenção dos 11 indicadores críticos desenvolvidos.

A escolha e aplicação da técnica Delphi possibilitou submeter o instrumento à apreciação de enfermeiros especialistas da área, com vivências distintas e valiosíssimas para o estudo; atendendo, portanto, aos motivos que nos levaram a optar por essa técnica.

O sistema de classificação desenvolvido e validado, atende, particular e exclusivamente, às necessidades de pacientes psiquiátricos internados em sistema integral de tratamento.

A quantidade de horas da assistência de enfermagem psiquiátrica poderá ser medida baseada na implementação deste sistema no cotidiano da prática hospitalar.

Enfim, acredita-se que com o desenvolvimento deste estudo tenhamos contribuído para o avanço tecnológico e científico da Especialidade Enfermagem Psiquiátrica e Saúde Mental.

\section{CONSIDERAÇÕES FINAIS}

As alterações propostas foram atendidas, porém, vale ressaltar que a validação clínica do instrumento será objeto de futuro estudo, devendo responder se pacientes denominados "graves" serão classificados como "nível de dependência plena" à enfermagem psiquiátrica.

A classificação do nível de dependência de pacientes psiquiátricos foi o primeiro passo para o dimensionamento de pessoal da especialidade em unidades de internação, devendo ainda em estudos posteriores determinar-se o cálculo de pessoal e a adequação da assistência de enfermagem.

\section{REFERÊNCIAS}

1. Souza MCBM, Alencastre MB. A formação de enfermeiro psiquiátrico e a pesquisa em enfermagem: algumas considerações teóricas. Acta Paul Enferm. 2003;16(1):76-83.

2. Fugulin FMT. Sistema de classificação de pacientes: análise das horas de assistência de enfermagem. [Dissertação Mestrado]. São Paulo: Escola de Enferm. da Universidade de São Paulo; 1997.

3. Rodrigues Filho J. Sistema de classificação de pacientes. Parte I. Dimensionamento de pessoal de enfermagem. Rev Esc Enfermagem USP. 1992; 26(3):395-404.

4. Alcalá MU, Nunes MF, Kato T, Reigada I, Silva RML. Cálculo de pessoal: estudo preliminar para estabelecimento de quadro de pessoal de enfermagem na superintendência médico-hospitalar de urgência. São Paulo: Secretaria Municipal da Saúde e Higiene; 1982.

5. Perroca MG. Sistema de classificação de pacientes: construção e validação de um instrumento [tese]. São Paulo: Escola de Enfermagem da Universidade de São Paulo; 1996.

6. Gaidzinski RR, Kurcgant P. Dimensionamento do pessoal de enfermagem: vivência de enfermeiros. Nursing (São Paulo).1998;1(2):28-34.

7. Giovannetti P. Understanding patient classification systems. J Nurs Adm. 1979; 9(2):4-9.

8. Giovannetti P, Maiyer GG. Building confidence in patient classification systems. Nurs Manage. 1984;15(8):31-4.

9. Santos SR. Cálculo de pessoal de enfermagem: estudo de dois métodos. Rev Esc Enferm. USP. 1992; 26(2):137-53.

10. Ramos MLT, Bohomol E, Santos M, Barros CG, Ferreira NM, Dornaus MFPS, et al. Sistema de 
classificação de pacientes por grau de dependência do cuidado de enfermagem: ENFSCORE. In: $5^{\circ}$ Encontro de Enfermagem e Tecnologia - ENFTEC; 1996; São Paulo. Anais. São Paulo: ENFTEC; 1996. p.128-41.

11. Romero EA, Araujo ES, Watanabe MY, Lisboa MAPLP, Abud MH, Barroso RV, et al. Proposta de um instrumento para a classificação dos cuidados de enfermagem quanto ao grau de dependência. In: $5^{\circ}$ Encontro de Enfermagem e Tecnologia - ENFTEC; 1996; São Paulo. Anais. São Paulo: ENFTEC; 1996. p. 482-5.

12. Gaidzinski RR. Dimensionamento de pessoal de enfermagem em instituições hospitalares. [tese]. São Paulo: Escola de Enfermagem da Universidade de São Paulo; 1998.

13. Kurcgant P, Cunha KC, Gaidzinski RR. Subsídios para a estimativa de pessoal de enfermagem. Enfoque São Paulo. 1989;17(3):79-81.

14. Gaidzinski RR. Dimensionamento de pessoal de enfermagem. In: Kurcgant P, coordenador. Administração em enfermagem. São Paulo: EPU; c1991. p. 91-6.

15. Ribeiro CM. Sistema de classificação de pacientes como subsidio para provimento de pessoal de enfermagem. [tese]. São Paulo: Escola de Enfermagem da Universidade de São Paulo; 1972.
16. Conselho Federal de Enfermagem (COFEn). Resolução n.189/96. Estabelece parâmetros para dimensionamento do quadro de profissionais de enfermagem nas instituições de saúde. In: Conselho Regional de Enfermagem de São Paulo (COREn$\mathrm{SP})$. Documentos básicos de enfermagem: enfermeiros, técnicos e auxiliares. São Paulo: COREnSP; 1997. p.177-80.

17. Rodrigues Filho J. Sistema de Classificação de Pacientes - parte II: custo de enfermagem. Rev Esc Enferm. USP. 1994; 28(1):41-9.

18. Morath J, Fleischmann R, Boggs G. A missing consideration: the psychiatric patient classification for scheduling-staffing systems. Perspect Psychiatr Care. 1990; 25(3-4):40-7.

19. Sims L, Kippenbrock TA. Psychiatric nurses' satisfaction with a patient classification system for staffing. Issues Ment Health Nurs. 1994;15(4):409-17.

20. Arantes EC. Observação de comportamento de pacientes internados em hospital psiquiátrico. Rev Bras Enferm. 1968;21(1):39-49.

21. Spínola AWP. Delfos: proposta tecnológica alternativa. São Paulo: Faculdade de Saúde Pública; 1984.

22. Faro ACM. Técnica Delphi na validação das intervenções de enfermagem. Rev Esc Enferm. USP. 1997; 31(2):259-73. 


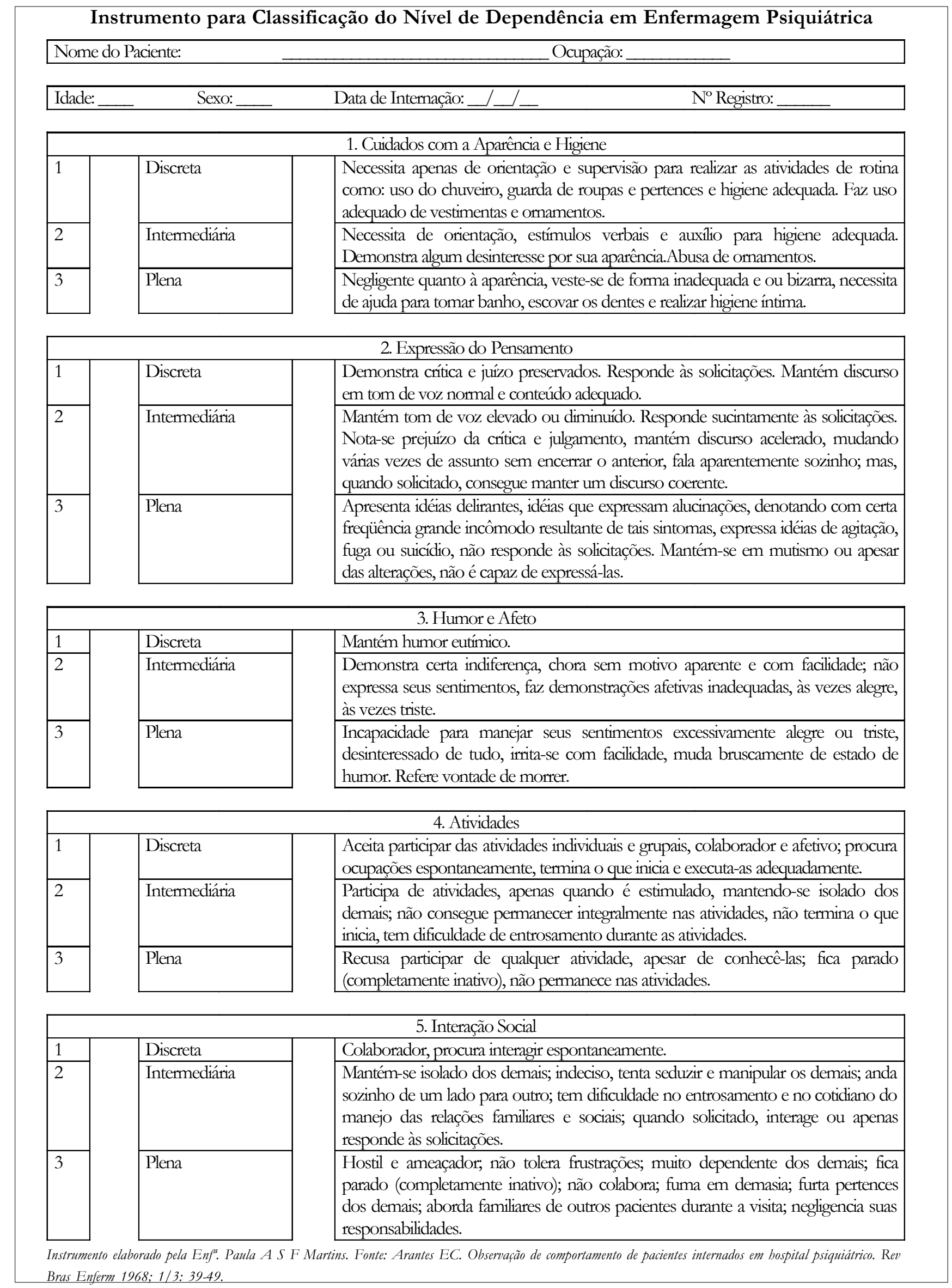




\begin{tabular}{|c|c|c|}
\hline \multicolumn{3}{|r|}{ 6. Alimentação / Hidratação } \\
\hline 1 & Discreta & $\begin{array}{l}\text { Aceita adequadamente as refeições e hidratação; mantém hábitos adequados durante } \\
\text { as refeições, considerando-se as diferenças culturais. }\end{array}$ \\
\hline 2 & Intermediária & $\begin{array}{l}\text { Ingere quantidade insuficiente de alimentos; exige dieta especial (terapêutica); } \\
\text { quando estimulado e orientado alimenta-se; mantém alguns hábitos inadequados } \\
\text { durante as refeições, considerando-se as diferenças culturais. }\end{array}$ \\
\hline 3 & Plena & $\begin{array}{l}\text { Não se alimenta sozinho; tem dificuldade para mastigar ou deglutir; recusa as } \\
\text { refeições; ingere quantidade excessiva de alimentos; mantém-se inadequado durante } \\
\text { as refeições; realiza ações purgativas, após as refeições. }\end{array}$ \\
\hline
\end{tabular}

\begin{tabular}{|l|l|l|}
\hline 1 & \multicolumn{2}{|l|}{} \\
\hline 2 & & Discreta \\
& & Intermediária \\
\cline { 1 - 1 } & & Plena \\
\hline
\end{tabular}

\section{Sono}

Dorme regularmente à noite.

Dorme durante o dia; não dorme à noite mas permanece em seu leito; só dorme após ser medicado (s/n).

Dorme e queixa-se de que não dormiu; não dorme dia e noite e torna-se inquieto e agitado; sonâmbulo; não dorme mesmo depois de medicado uma vez; dorme além do normal tanto de dia como à noite.

\begin{tabular}{|c|c|}
\hline 1 & Discreta \\
\hline 2 & Intermediária \\
\hline 3 & Plena \\
\hline
\end{tabular}

\section{Medicação}

Aceita sua medicação; quase sempre conhece os medicamentos que usa, bem como seus efeitos; é possível responsabilizá-lo pela própria medicação.

Aceita sua medicação após orientação e abordagem; apresenta sintomas de efeitos colaterais e indesejáveis da medicação; desconhece os medicamentos que usa, bem como seus efeitos; demonstra certa insatisfação ou medo dos medicamentos; eventualmente, procura por informações sobre a medicação.

Faz tentativas de esconder sua medicação; recusa os medicamentos; necessita de medicações injetáveis; solicita medicamentos a todo o momento.

\begin{tabular}{|c|c|c|}
\hline \multicolumn{3}{|r|}{ 9. Eliminações } \\
\hline 1 & Discreta & As eliminações estão presentes; tem controle esfincteriano. \\
\hline 2 & Intermediária & $\begin{array}{l}\text { Suas eliminações não são diárias ou são excessivas; tem controle esfincteriano; } \\
\text { apresenta obstipação ou eliminações intestinais líquidas; apresenta incontinência } \\
\text { urinária decorrente do uso de medicações. }\end{array}$ \\
\hline 3 & Plena & Não tem controle esfincteriano; faz uso inadequado do sanitário. \\
\hline
\end{tabular}

\begin{tabular}{|c|c|c|}
\hline \multicolumn{3}{|r|}{ 10. Sinais Vitais e outros Controles } \\
\hline 1 & Discreta & Necessita de verificação sistematizadamente. \\
\hline 2 & Intermediária & $\begin{array}{l}\text { Necessita de verificações de acordo com a evolução clínica, sintomatológica ou } \\
\text { queixas. }\end{array}$ \\
\hline 3 & Plena & $\begin{array}{l}\text { Necessita de controle de sinais vitais, hídrico, de débito urinário, glicemia, ou } \\
\text { outros, várias vezes ao dia; apresenta disfunções clínicas não psiquiátricas (HAS; } \\
\text { Diabetes Mellitus; outras). }\end{array}$ \\
\hline
\end{tabular}

\begin{tabular}{|c|c|c|}
\hline \multicolumn{3}{|r|}{ 11. Queixas e Problemas Somáticos } \\
\hline 1 & Discreta & Nega queixas somáticas. \\
\hline 2 & Intermediária & \begin{tabular}{|l}
$\begin{array}{l}\text { Refere queixas relativas ao tratamento medicamentoso, de sinais e sintomas } \\
\text { crônicos ou outras. }\end{array}$ \\
\end{tabular} \\
\hline 3 & Plena & $\begin{array}{l}\text { Refere queixas de sintomas agudos de disfunções fisiológicas ou clínicas; apresenta } \\
\text { sinais e sintomas de patologias clínicas. }\end{array}$ \\
\hline
\end{tabular}

\begin{tabular}{|ll|l|l|}
\hline Total: & Pontos & Classificação - Nível de Dependência & \\
\hline
\end{tabular}

\begin{tabular}{|c|l|}
\hline Diagnóstico de Enfermagem: \\
Diagnóstico Clínico:
\end{tabular}

